\title{
Autoregressive models as a tool to discriminate chaos from randomness in geoelectrical time series: an application to earthquake prediction
}

\author{
Vincenzo Cuomo $\left({ }^{1}\right)$, Vincenzo Lapenna $\left({ }^{1}\right)$, Maria Macchiato $\left({ }^{2}\right)$ and Carmine Serio $\left({ }^{3}\right)$ \\ $\left({ }^{1}\right)$ Istituto di Metodologie Avanzate di Analisi Ambientale, IMAAA/CNR, Tito Scalo $(P Z)$, Italy
$\left({ }^{2}\right)$ Dipartimento di Scienze Fisiche, Università Federico II, Napoli, Italy
$\left.{ }^{3}\right)$ Dipartimento di Ingegneria e Fisica dell'Ambiente, Università della Basilicata, Potenza, Italy \\ $\left({ }^{2}\right)$ Dipartimento di Scienze Fisiche, Università Federico II, Napoli, Italy
$\left({ }^{3}\right)$ Dipartimento di Ingegneria e Fisica dell'Ambiente, Università della Basilicata, Potenza, Italy
}

\begin{abstract}
The time dynamics of geoelectrical precursory time series has been investigated and a method to discriminate chaotic behaviour in geoelectrical precursory time series is proposed. It allows us to detect low-dimensional chaos when the only information about the time series comes from the time series themselves. The short-term approximation and local autoregressive app using two possible forecasting approaches: global autoregressive stochastic process, whereas the second considers supposedly non-linear. The comparison of the predita points as a realization of a deterministic process, between low-dimensional chaos and rando predictive skill of the two techniques is a test to discriminate ments recorded by an automatic station located inamics. The analyzed time series are geoelectrical measureMediterranean region. Our finding are physical system governing the global (linear) approach is superior to the local one and the freedom. Power spectra of the filtered tima of electrical nature is characterized by a large number of degrees of behaviour of a broad class of fractal stochastic follow a $P(f)=f^{-\alpha}$ scaling law: they exhibit the typical systems.
\end{abstract}

Key words self-potential time series - chaos dynamical systems - earthquake prediction

\section{Introduction}

Geoelectrical time series have been studied as precursors of seismic events by many authors. In past years some strong events in China (Raleigh et al., 1977) have been forecasted using geoelectrical measurements, some

Mailing address: Dr. Vincenzo Cuomo, Istituto di Metodologie Avanzate di Analisi Ambientale, IMAAA/ CNR, Tito Scalo (PZ), Italy; e-mail: cuomo@unibas.it networks for systematic observations have been tested in Greece (Varotsos and Alexopoulos, 1984a,b; Varotsos et al., 1993) and along Crete Island (Nomikos et al., 1994) to predict local earthquakes and intensive studies to detect resistivity variations were carried out in the Parkfield experiment (Park and Fitterman, 1990).

On the other hand, it has been well demonstrated, using laboratory tests and/or outdoor controlled experiments, that an increased stress field over rock prisms modifies the resistivity and produces self-potential transient signals (Joanoiux et al., 1994; Hadjicontis and Mavromatou, 1994; Sornette and Sornette, 1990). 
However, the use of the electrical precursors in earthquake prediction is, to a large extent, still empirical due to the many difficulties that continue to exist in anderstanding the physics that underlies the problem (Rikitake, 1988). Recently many authors carried out works (Teisseyre, 1997; Eftaxias et al., 1993; Nishizava et al., 1994) to remove the ambiguity in the application of electrical precursors. In particular, the mean weak point of this deterministic approach to the earthquake prediction problem is the absence of a complete robust statistical evaluation of the efficiency of the electrical precursors (Burton, 1985; Mulargia and Gasperini, 1992).

For this reason, the statistical analysis of the anomalies in the geoelectrical time series is a very important task when we approach the earthquake prediction problem. A firm quantitative methodology is necessary to select anomalous patterns from the background noise, but the anomalies can be correctly detected only when the time evolution of the electrical signal is known.

To fill this gap we investigated the time dynamics (i.e., deterministic components, stochastic nature, chaotic behaviour) of these processes, studying in particular the short-term predictability of the geoelectrical time series. Our findings will give information on the statistical features of the geoelectrical background noise and of the dynamical system producing the electrical phenomena observed on the earth surface in seismic areas.

This paper discusses the statistical analysis of dynamical systems based on the estimation of their degree of predictability. The analysis can distinguish randomness from chaos and can provide a parsimonious representation in terms of autoregressive models of observations. These models may be used to forecast future values of the phenomenon. The analysis applies to a single scalar observable or scalar time series of a given observable and can give information on the number of degrees of freedom that are relevant to the dynamics of the system.

The approach we propose consists in fitting autoregressive processes to the data, then forecasts can be produced on the basis of the se- lected model. We distinguish between two possible strategies of approximating a given time series with autoregressive processes. The global autoregressive technique views the signal as the realization of a stochastic process: the autoregressive model is fitted to all the data points at once and estimates of the autoregression coefficients are computed consistently with the above assumption of randomness. On the other hand, based on recent ideas about the problem of forecasting chaotic time series (Farmer and Sidorowich, 1987; Casdagli 1992; Serio 1992, 1994a,b; Cuomo et al., 1994), the local autoregressive technique assumes the signal to be truly deterministic and the autoregression coefficients, and consequently predictions, are obtained according to such an assumption.

In practice, we compare the forecast error functions computed according to the two techniques. Since two such functions obey different scaling laws, their proper comparison allows us to gain insight into the knowledge of the dynamical characteristics of the process.

Non-linear short-term predictability as a tool to distinguish chaos from randomness dates back to Farmer and Sidorowich (1987). The topic was been later discussed by Sugihara and May (1990), Casdagli (1992), Kennel and Isabelle (1992) and Tsonis and Elsner (1992). A possible drawback of non-linear short term prediction is that random systems (e.g., Fractional Brownian Motion) may exhibit the same characters (correlation, covariance, predictability, scaling properties) as chaotic systems. Our method overcomes such a difficulty by a proper intercomparison between the predictive skills of linear (global) and non-linear (local) predictors.

The second goal of this paper is to give an estimate of the number of degrees of freedom governing the time evolution of the dynamical system under study. In fact, studying the shortterm predictability of the self-potential time series we have information on the dynamical process responsible for the observed electrical anomalies. When the forecasting gives better results using a local approach, the degrees of freedom of the system are few, otherwise if the 
global approach proves better we have an infinite dimensional system.

On the basis of the results obtained from the autoregressive analysis of the signal, we also explored the possibility to apply the recent methodologies regarding Self-Organized Criticality (SOC) (Bak et al., 1988) to the analysis of precursory phenomena.

The basic idea underlying our approach is that the lithosphere can be viewed as an extended dissipative system; recently it has been demonstrated that these systems naturally evolve into a self-organized critical state (states without detailed specification of the initial conditions) (Turcotte, 1992). The tectonics of the Earth and all the unexplained correlations of the geophysical and the geochemical field over the earth surface can be considered a sequence of instability episodes and earthquakes are the natural consequences of the dynamical state of the crust submitted to steady increasing stresses. The temporal fingerprint of the self-organized critical state is the presence of the $1 / f$ noise and the spatial signature is the emergence of scale invariant (fractal) structure (Bak et al., 1988).

To this purpose a thorough analysis of the spectral features of geoelectrical time series was been performed. The second goal of this work is the detection of behaviour in the power spectra typical of $1 / f$ noises.

Finally, an analysis of the implications of the earthquake prediction problem will be discussed applying our methods on the geoelectrical data collected in Tito (Southern Italy).

\section{Data}

In this work we use the geoelectrical time series recorded by an automatic station located in Southern Italy (fig. 1). Since May 1991 two arrays have been installed using copper electrodes put in the ground at $1 \mathrm{~m}$ depth along the $\mathrm{N}-\mathrm{S}$ and E-W directions, spaced $100 \mathrm{~m}$ and $120 \mathrm{~m}$ respectively, near the Area della Ricerca of the National Research Council located in Tito (PZ) $\left(40^{\circ} 35^{\prime} \mathrm{N}, 15^{\circ} 44^{\prime} \mathrm{E}\right)$. Technically a geoelectric or self-potential time series is a sequence of voltage differences measured with a

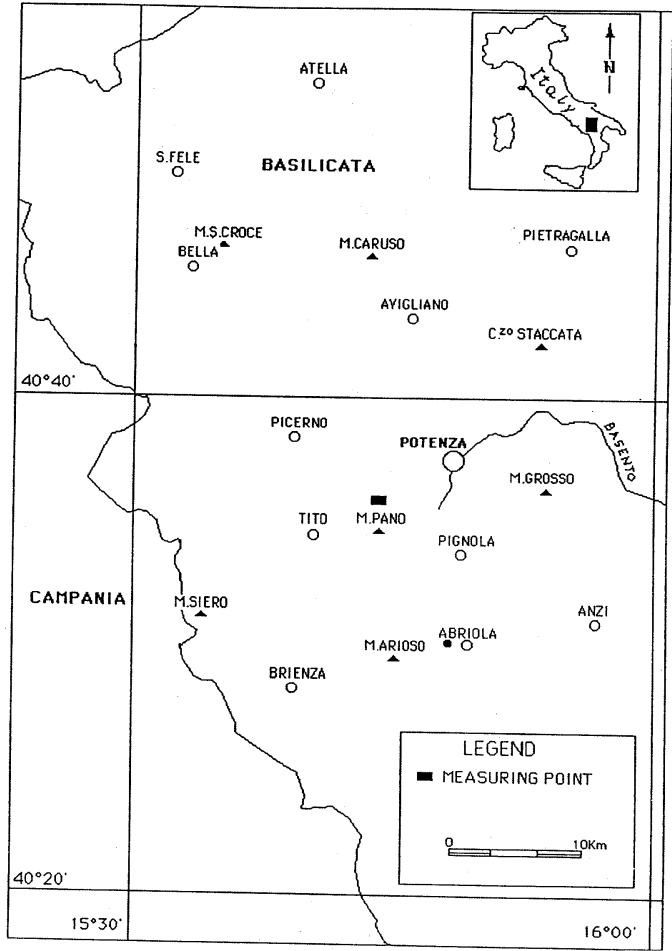

Fig. 1. Location of the automatic station devoted to the measurement of self-potential signals.

selected sampling interval using a receiving electrode array. During the geoelectrical soundings, where a current is injected into the ground, the self potential represents the noise (Lapenna et al., 1994). On the other hand, when we record using a passive measurement technique (i.e., without energizing system), for example in a seismic area, it is the signal.

In the Tito station a multi-channel data-logger is equipped to measure the voltage differences between the probes and also some meteoclimatic parameters (temperature, humidity, radiance, etc.). The sampling rate is $\Delta t=30 \mathrm{~s}$, the acquisition procedures are controlled by a personal computer connected to the data-logger that computes and stores the mean voltage values every $5 \mathrm{~min}$. The daily values of self-potential data recorded during the period May 1991 - April 1994 are reported in fig. 2. 

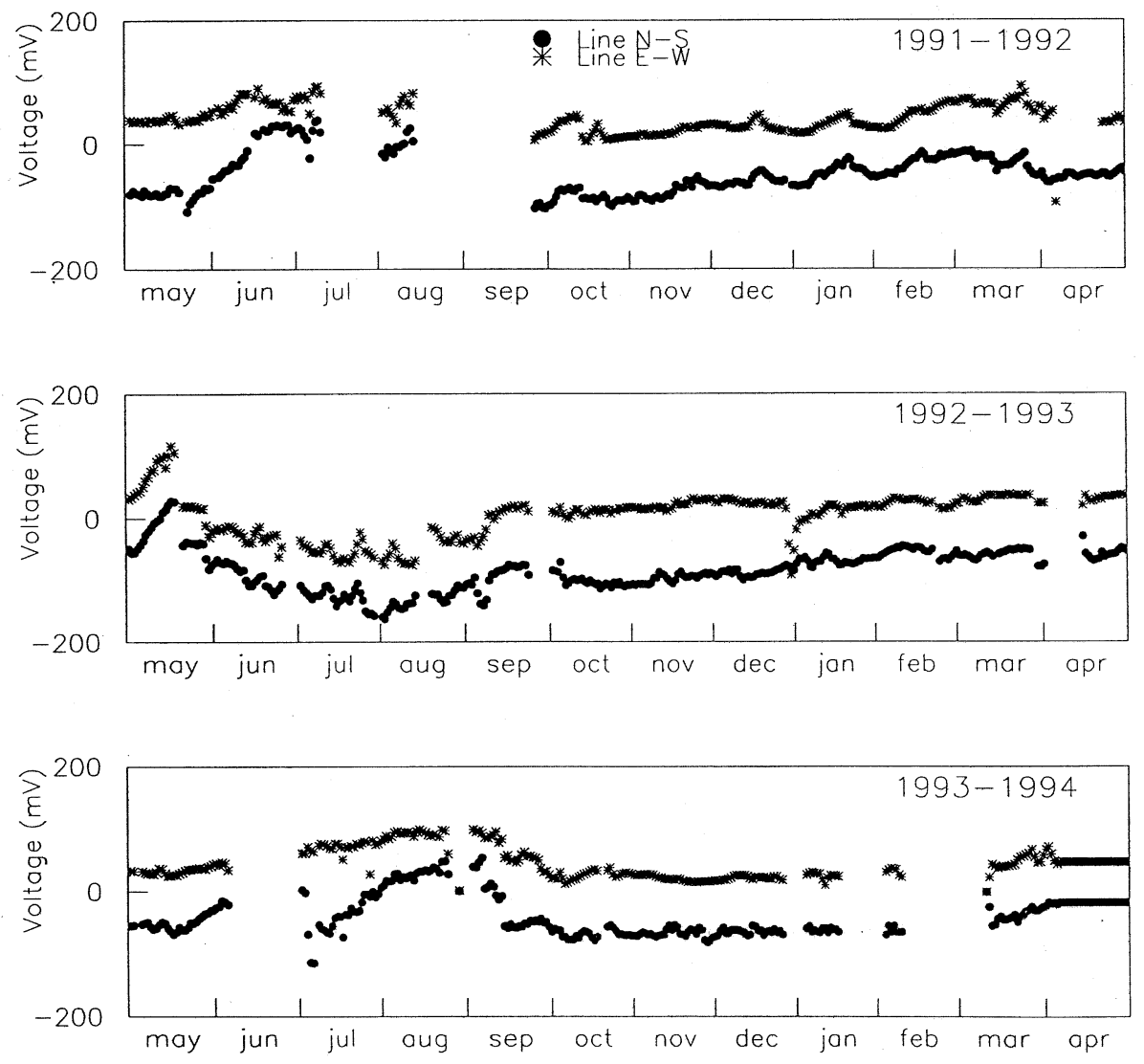

Fig. 2. Daily self-potential time series recorded by Tito station.

We had a great number of data missing only during the period July 1991 - September 1991 because we upgraded the station, while there are no other periods with a high number of data missing, the global ratio, between missing and measured values, being $10 \%$. To avoid some self polarizing effects we used ceramic electrodes built of ceramic vessels filled with a saturated solution of copper sulphate. The time series obtained with the different probes are constantly checked to remove anomalous patterns related to polarizable effects (Di Bello et al., 1994).

Furthermore, the self-potential measurements can be influenced by many geophysical parameters (i.e., seasonal effects of the climatological variables, magnetic storms etc.), so a preliminary filtering procedure is necessary to remove these effects on the data (Lapenna et al., 1994). Before going on we studied the correlation between the self-potential time series and the air temperature and the humidity time series recorded by the automatic station in Tito. For the sake of brevity we do not report in this paper the routinely filtering procedure, but all the analyzed data were preliminary filtered from any meteo-climatic effects. A firm quantitative analysis of the techniques devoted to the filtering of geoelectrical data is described in a previous paper (Di Bello et al., 1996). 
The measuring station is located on the Southern Apennine chain which is one of the most seismically active areas of the Mediterranean region. It is the result of a complex sequence of tectonic events associated with the collision between Africa and Europe. In this area on November 23, 1980, a large normalfaulting earthquake occurred. It was one of the largest event observed in Italy in the past 80 years (Pantosti and Valensise, 1990).

In particular, the measuring station is located $7 \mathrm{~km}$ away from the town of Potenza, an area characterized by a local seismicity with intensities no larger than VII. In fact this seismic activity is related to a faulting system transverse to the great Apennine faults (Boschi et al., 1994) and these tectonic structures typically are responsible for non destructive earthquakes. The spatial pattern of local seismicity during the measuring period is depicted in fig. 3 .

In the investigated area there are favourable conditions to observe precursory phenomena of an electrical nature. Furthermore many rocks,

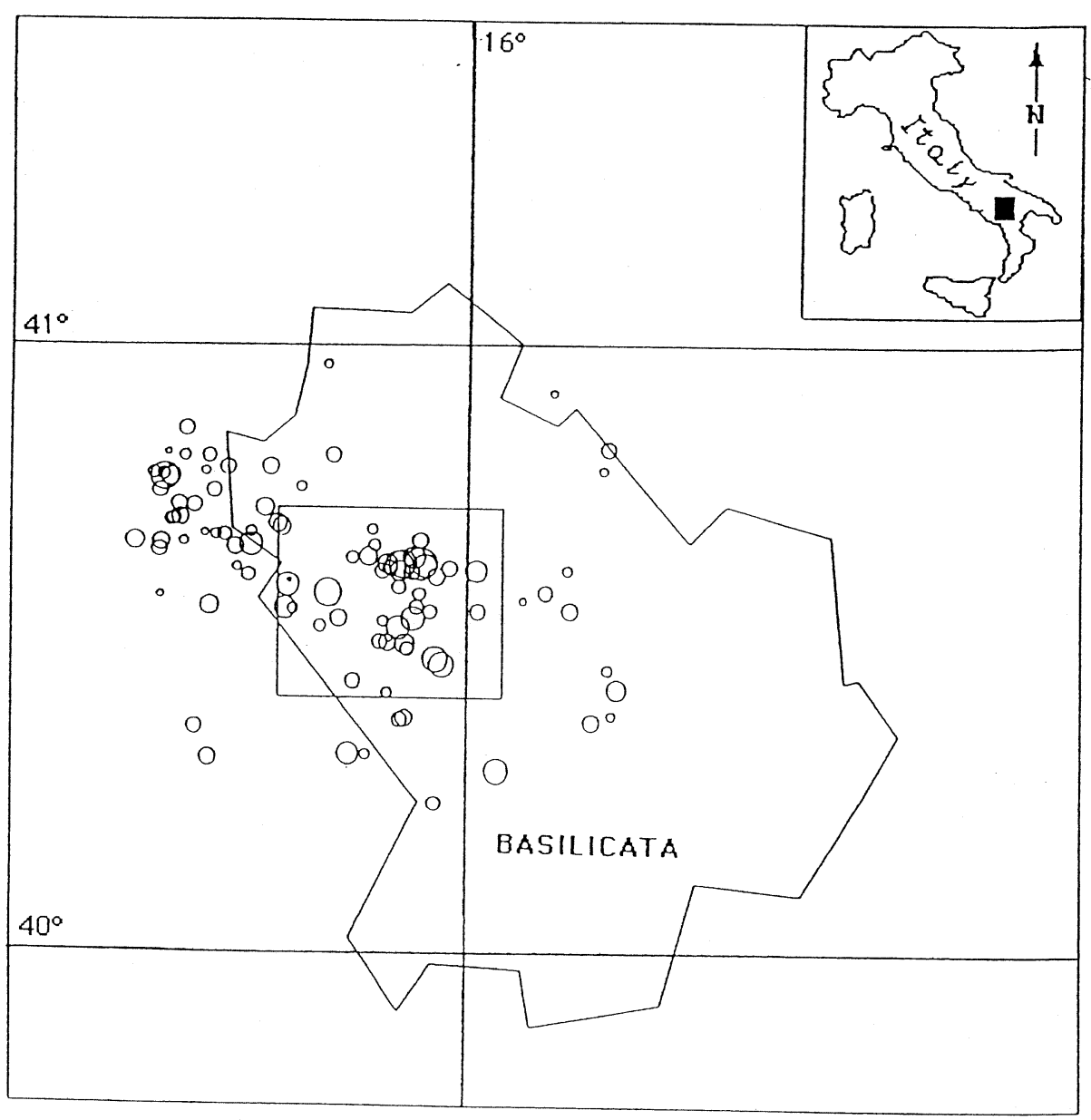

Fig. 3. Seismicity pattern in the monitored area of the Southern Apennines chain. The amplitude of the circles is related to the magnitude of the earthquakes varying in the range from $M=2$ to $M=5$. 
deep fluids and gases interact as recognized by recent investigations (Balderer and Martinelli, 1994), so it is possible to detect electrical anomalies possibly related to electrokinetic phenomena.

\section{Autoregressive techniques: global and local approaches}

To study the time dynamics of the geoelectrical time series recorded by the Tito station we use a methodology based on autoregressive processes. The rationale behind the scheme is that if only a few degrees of freedom interact non-linearly to generate deterministic chaos than a local (non-linear) predictor can be constructed which approximates the dynamics of the low-dimensional signal better than any global (linear) predictor. Technically, predictors are built by locally and globally fitting autoregressive processes to the data.

The global approach is equivalent to representing the observations by means of a linear (infinite-dimensional) stochastic model, whereas the local approach is equivalent to representing the data on the basis of a low-dimensional deterministic chaotic system. In this work, the predictive skill of the two approaches is compared. If the local representation gives a pre-dictive advantage over the global one, and therefore over the entire class of linear stochastic systems including regular attractors and coloured noise, the conclusion is for chaos, otherwise the reverse conclusion is taken.

Let $x(t)$ denote the function of time. We shall assume that the signal is sampled at equal intervals, say $\Delta t$, then writing $x(t=n \Delta t)=$ $x(n)$, the sequence $x(n),(n=1, \ldots ., N)(N$ being the number of data points) constitutes a discrete time series. In the global autoregressive representation the $l$-step ahead forecast $(l>1) \hat{x}(n+l)$ of $x(n+l)$, standing at origin $n$, is given by a linear function of current and previous observations i.e.:

$$
\begin{gathered}
\hat{x}(n+l)=\phi_{1}(l) x(n)+\phi_{2}(l) x(n-1)+ \\
\ldots . .+\phi_{p}(l) x(n+1-p)
\end{gathered}
$$

with $p$ the order of the filter and with the coefficients, $\phi_{1}(l), \phi_{2}(l), \ldots . ., \phi_{p}(l)$, are unknown to be determined.

To this end, we use the well known YuleWalker estimation procedure (e.g., Box and Jenkins, 1976). Note that these coefficients depend on $l$ but they do not depend on the time origin, that is, they are time-shift invariant. This is a consequence of the linear structure of the stochastic process with which the data points are modelled in the global representation (e.g., Box and Jenkins, 1976). Reflecting the infinite dimensionality of the system, the quality of predictions increases with the number of data used to obtain estimates of the autoregression coefficients.

Conversely, in the local autoregressive representation, to obtain the $l$-step ahead forecast, $(\hat{x}(n+l) ; l>1)$, starting at the origin $n$, we again construct a predictor which is an autoregressive filter:

$$
\begin{gathered}
\hat{x}(n+l)=\phi_{1}(n, l) x(n)+\phi_{2}(n, l) x(n-1)+ \\
\ldots . .+\phi_{p}(n, l) x(n+1-p)
\end{gathered}
$$

but now the autoregression coefficients, $\phi_{1}(n, l), \phi_{2}(n, l), \ldots, \phi_{p}(n, l)$, depend on the time origin $n$ and on $l$ as well. A strategy to compute the autoregression coefficients for the local case has been discussed by Serio (1992, 1994a). The key point of the local approach is the ability to find local portions of a given time series in the past which closely resemble the present, and base the forecasts on what happened immediately after these past events. Technically, we consider $k p$-ple,

$$
\begin{gathered}
x_{p}\left(m_{i}\right)=\left(x\left(m_{i}\right)\right), \ldots ., x\left(m_{i}-p+1\right) ; \\
m_{i}<n \text { and } i=1, \ldots ., k,
\end{gathered}
$$

that minimize the Euclidean norm $\| x_{p}(n)-$ $-x_{p}\left(m_{i}\right) \|$.

Then, we regard every $p$-ple $x_{p}\left(m_{i}\right)$ as the input of the filter (3.2) and $x\left(m_{i}+l\right)$ as the output. Finally the coefficients, $\phi_{1}(n, l), \phi_{2}(n, l)$, $\ldots, \phi_{p}(n, l)$, are obtained by best-fitting the autoregressive model to the couples: $x_{p}\left(m_{i}\right)$, $x(m+l)$. It should be noted that, for a given 
origin $n$, the search for the $k p$-ples is done once, while the best-fitting procedure is performed $l$ times, that is at each step $l$, in order to compute the $\phi_{j}(n, l)$. We apply the leastsquares method by singular-value decomposition (e.g., Press et al., 1986). When $k=p$ this is equivalent to linear interpolation which is notoriously unstable, therefore to ensure stability we choose $k=2 p$.

To evaluate the predictions we compute the forecast error for the $l$-step ahead prediction by: $e(l)=x(n+l)-\hat{x}(n+l)$, then the normalized root mean square forecasting error, $E(l)$, will be defined as the expectation value of the forecast error

$$
E^{2}(l)=\frac{\left\langle e^{2}(l)\right\rangle}{\sigma_{x}^{2}}
$$

where the angular brackets denote expectation and $\sigma_{x}$ is the standard deviation of the series. $E(l)$ provides a quantitative measure of the predictive skill of each representation (local or global).

In practice we divide the time series into two separate parts: a fitting set $x(1), \ldots, x\left(N_{1}\right)$ and a testing set $x\left(N_{1}+1\right), \ldots, \quad x\left(N_{1}+N_{2}\right)$, $N=N_{1}+N_{2}$ being the size of the sequence. For each given order $p$, the first part of the series is used to compute the autoregression coefficients (global or local), then forecasts are produced at the location of the data points in the second part of the series and the corresponding errors, $e_{p}(l)$ computed. Here, the subscript $p$ recalls that this operation is repeated for different values of $p$, i.e., $p=1, \ldots ., p_{u}$, where $p_{u}$ is an upper bound specified from the user. Then, the forecast error function $E p(l)$ is computed and the norm, $K(p)$, evaluated by

$$
K(p)=\sqrt{E_{p}^{2}(l)+\ldots .+E_{p}^{2}\left(l_{\max }\right)}
$$

Finally, we adopt as the order of the process, $p_{\text {opt }}$, that $p$ for which $K(p)$ is minimized. Here, $l_{\max }$ is the maximum step ahead at which forecasts are obtained. In practice, it is obtained by the condition $E\left(l_{\max }\right)=1$.

The procedure is used both in the case of the global predictors and in the case of the local ones. In any case we obtain true optimal mean-square predictors. This point is important since for arbitrary $p$ the representation (3.1) is not necessarily the best in the least-square sense.

Provided that the signal $x(t)$ is the output of a chaotic system and $p>D$, where $D$ is the dimension of the underlying attractor, it can be demonstrated (Serio, 1994b) that

$$
E_{g l}(l)>E_{l c}(l)
$$

where $E_{g l}(l)$ denotes the root mean square forecasting error affecting the global prediction and $E_{l c}(l)$ is the corresponding value in the case of the local prediction.

This property can be easily demonstrated on the basis of the Kolmogorov linear mean square estimation theory. A stochastic process admits only a limited predictability, while a chaotic signal generated by a non-linear set of differential equations is continuous and differentiable and it has an exact local prediction (i.e., the equations are locally linearizable). Conversely, if the signal is stochastic the reverse of eq. (3.6) is expected since the global representation takes better into account the infinite dimensionality of the process (Drahos et al., 1995).

\section{Results}

In this section a thorough analysis of the time dynamics of the geoelectrical time series has been performed using the above mentioned methods. This study is supported by a spectral analysis of the signals and all the results allow us to improve the knowledge about the physical process underlying the observed electrical phenomena. Finally, in the last sub-section, all the implications on the earthquake prediction problem are analyzed.

\subsection{Detecting low-dimensional chaos}

The Global Autoregressive (GA) and Local Autoregressive (LA) techniques have been applied to discriminate low-dimensional chaos in geoelectrical time series at different - time scales. 
Firstly we investigated self-potential data over a large time scale. From the observed data, one sample every $5 \mathrm{~min}$, the daily mean values were obtained. In this way two daily self-potential time series were analyzed, the first one obtained from the dipole oriented in the $\mathrm{N}-\mathrm{S}$ direction and the second one from the dipole oriented in the E-W. The autoregressive techniques, GA and LA, were applied using the algorithms described in the previous section and the mean square forecast errors, $E^{2}(l)$, are plotted in fig. 4a,b. The global approach was better than the local one, so the self-potential time series can be well described by a stochastic process. Our findings confirm the results discussed in a recent paper (Cuomo et al., 1996) where an autoregressive process of order 2 was selected to describe daily self-potential time series. According to the global approach the estimates of parameters of the autoregressive model fitted to the data increase with the number of data available.

At this point, we explore the data at a very short time scale sampling interval, the entire set (sampling interval $\Delta t=5 \mathrm{~min}$ ) is subdivided into sub-samples, each built up with 2880 consecutive values, and for each record we estimated the mean square forecast errors
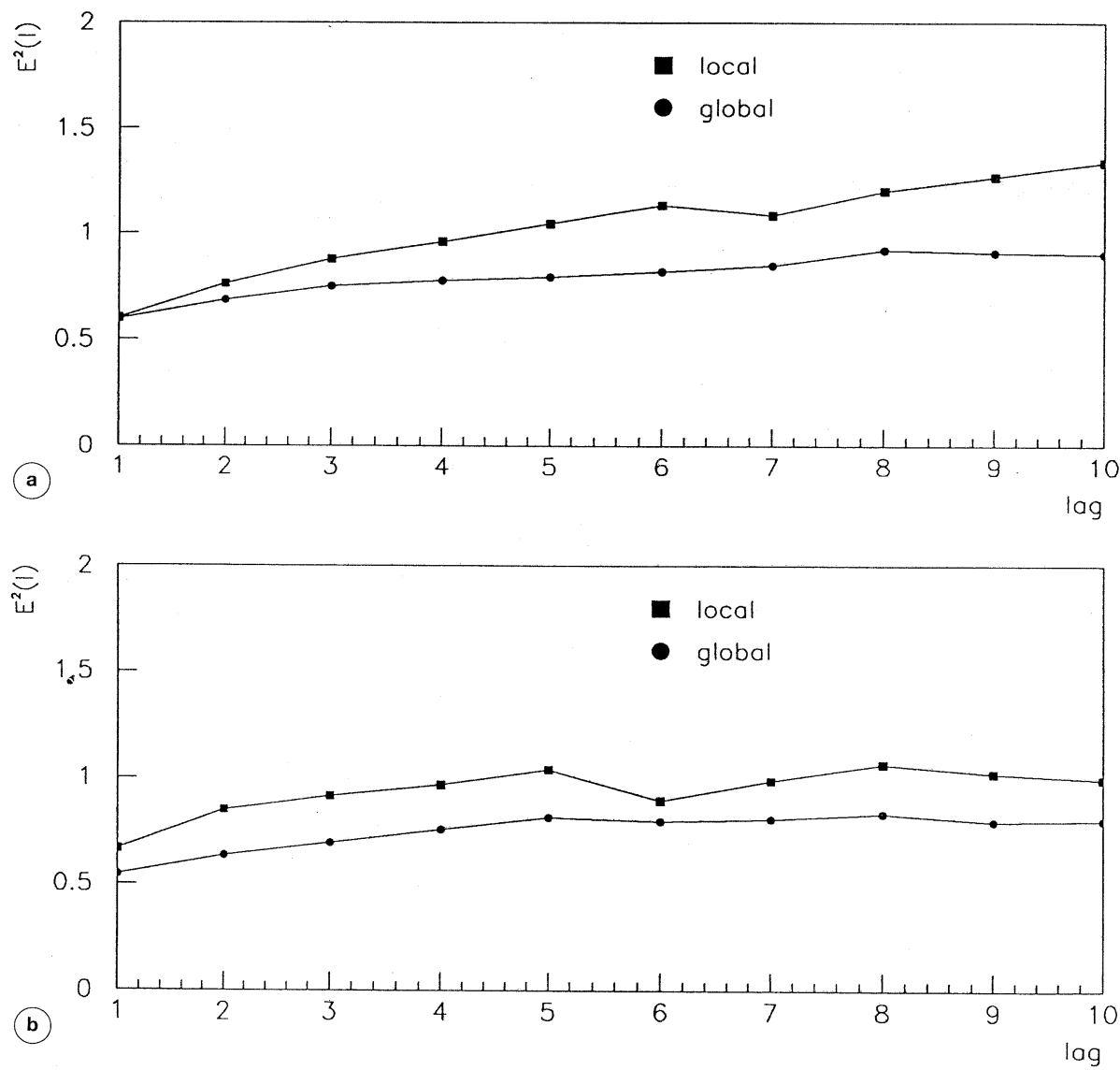

Fig. 4a,b. Mean square forecast errors obtained from the analysis of daily self-potential time series. Figure $4 \mathrm{a}, \mathrm{b}$ reports the results regarding the data obtained from the measuring dipoles oriented in N-S and E-W directions. 

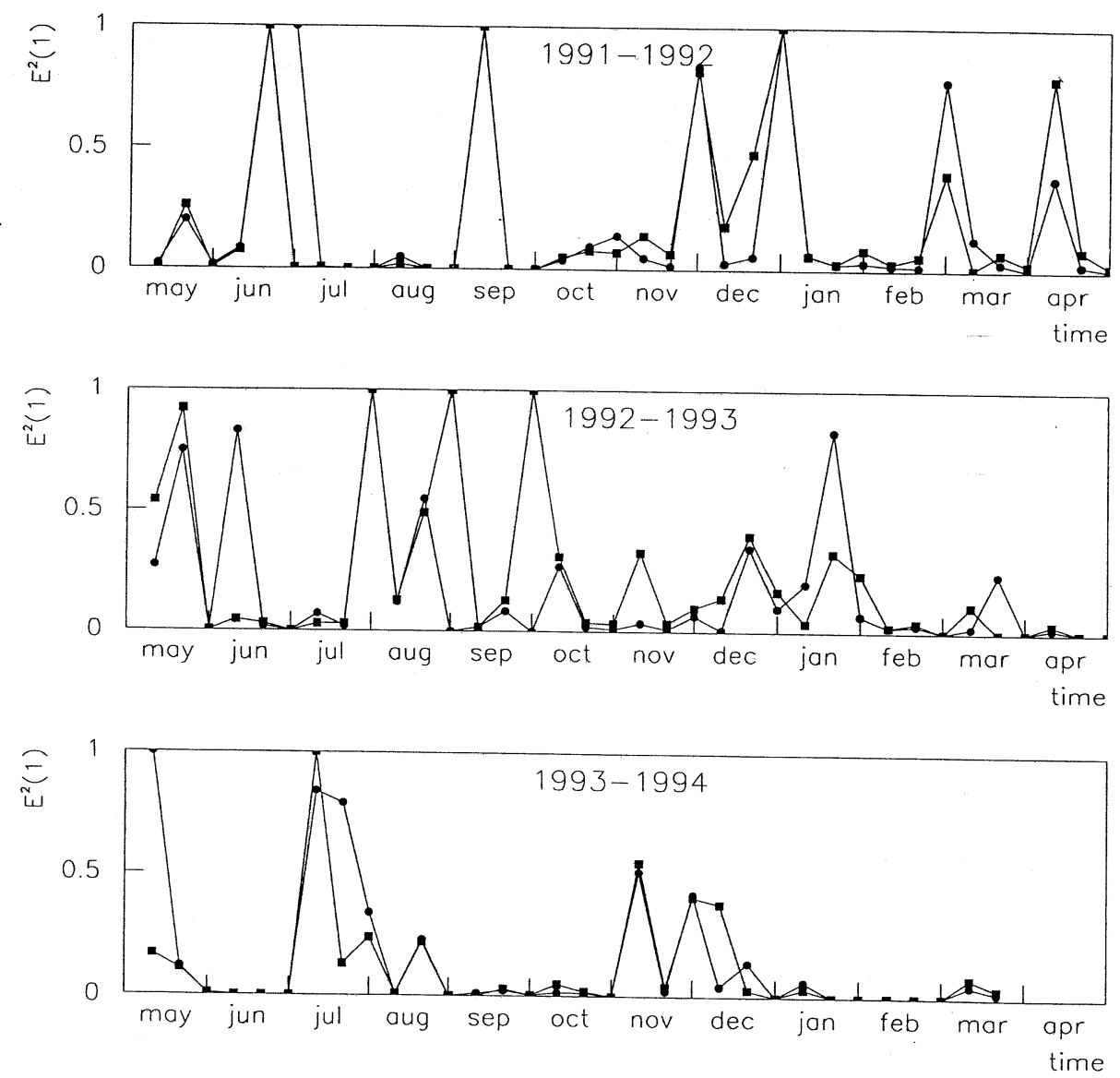

Fig. 5. Mean square forecast errors, with $l=1$, obtained applying the global and local techniques to sub-sets of the self-potential time series (line E-W). Each sub-set is built up with 2880 data points.

using the GA and LA techniques for data from May 1991 to April 1994. The graphs of the estimates of $E^{2}(l)$ versus time are plotted in fig. 5 . The data processed were obtained using the dipole oriented in the E-W direction. Observing the results of the forecast errors during the three years, we can exclude at a very short time scale the presence of low-dimensional chaos, since the global and local approaches always give comparable results. The physical process that produces the geoelectrical phenomena is described by an infinite dimensional dynamical system.

Our findings suggest including the geoelectrical time series in a wide class of Fractal
Brownian Motion (FBM). In fact, the curves $E^{2}(l)$ for the global case are very stable which demonstrates the presence of correlations also at lead time $l$ as large as 10 and long range correlations are typical of FBM processes (Voss, 1989).

To better substantiate our results a comparison with a method proposed by Tsonis and Elsner (1992) was analyzed. In this article the authors pointed out that Pearson's correlation coefficient, $r$, between predicted and actual values decreases exponentially with the prediction time when a chaotic signal is present. On the other hand, when we have FBMs the expo- 
nentially behaviour is absent. On these basis they proposed a method to discriminate lowdimensional chaos in experimental time series.

In fig. 6, as described in the paper of Tsonis and Elsner, the $(1-r)$ values versus the prediction time lag are reported on log-log scales. According to the results obtained using our approach, the linear behaviours of the curves in fig. 6 are typical fingerprints of FBM signals.

This agreement is dictated by the theoretical relation between the forecast error and the correlation coefficient (Wales, 1991):

$$
r=1-\frac{\sigma^{2}\left(x_{i}-y_{i}\right)}{2 \sigma^{2}}
$$

where $r$ is the correlation coefficient, $\sigma^{2}$ the sampling variance of data and $\sigma^{2}\left(x_{i}-y_{i}\right)$ the forecast error (in our text reported $E^{2}(l)$ ).

\subsection{I/f noises and self-organized systems}

In this sub-section the main features of the geolectrical time series in the frequency domain are analyzed. Recalling some recent papers (Bak et al., 1988; Sornette and Sornette, 1989) concerning the complex dynamics of extended systems with a very large number of degrees of freedom, we studied the power spectra of geoelectrical time series. In fact, typical fingerprints of these systems are the flicker or $1 / f$ noises, so the power spectra of
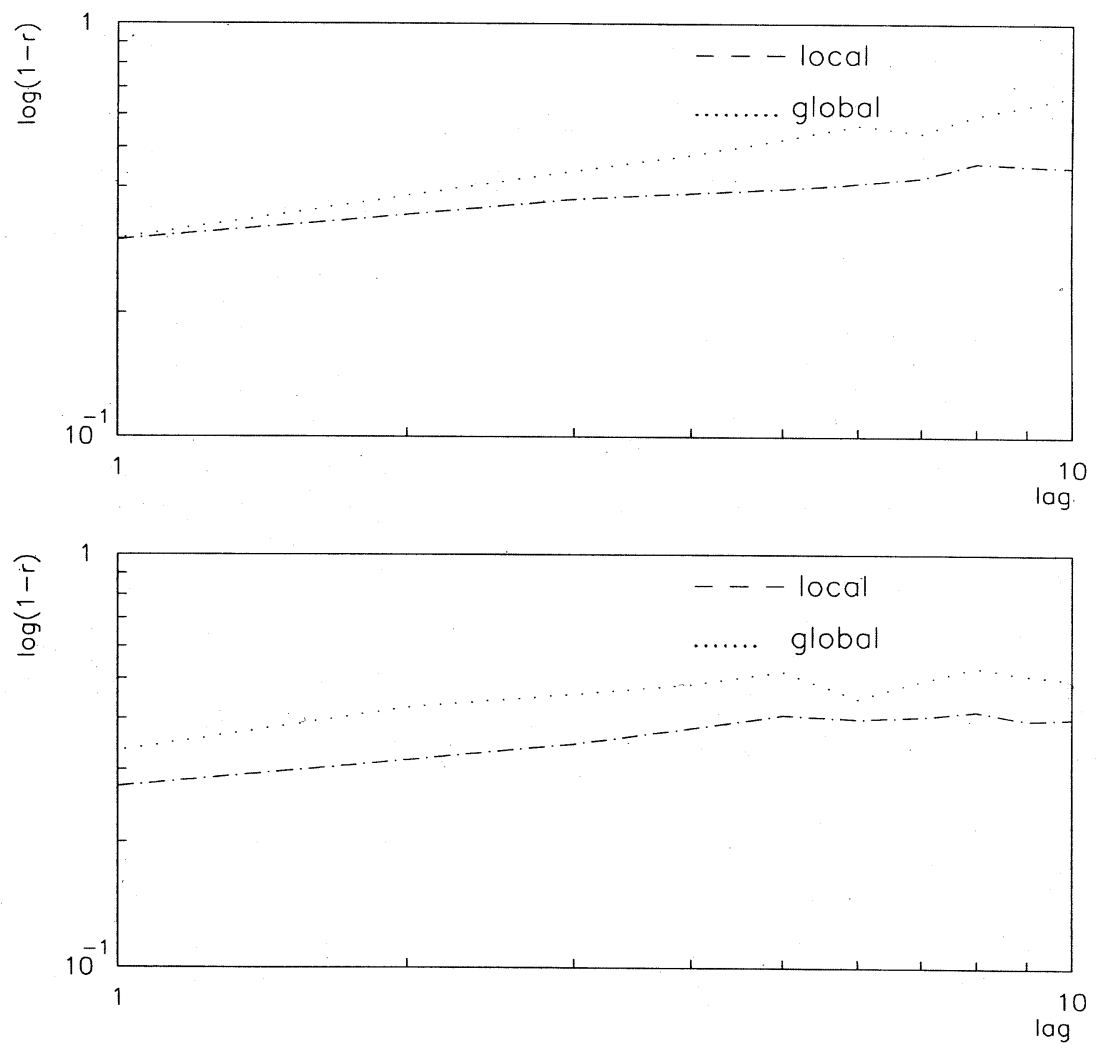

Fig. 6. $1-r$ values $v s$. prediction time lag on $\log -\log$ scales. Linear behaviour is observed in the plot as expected for an FBM signal. 

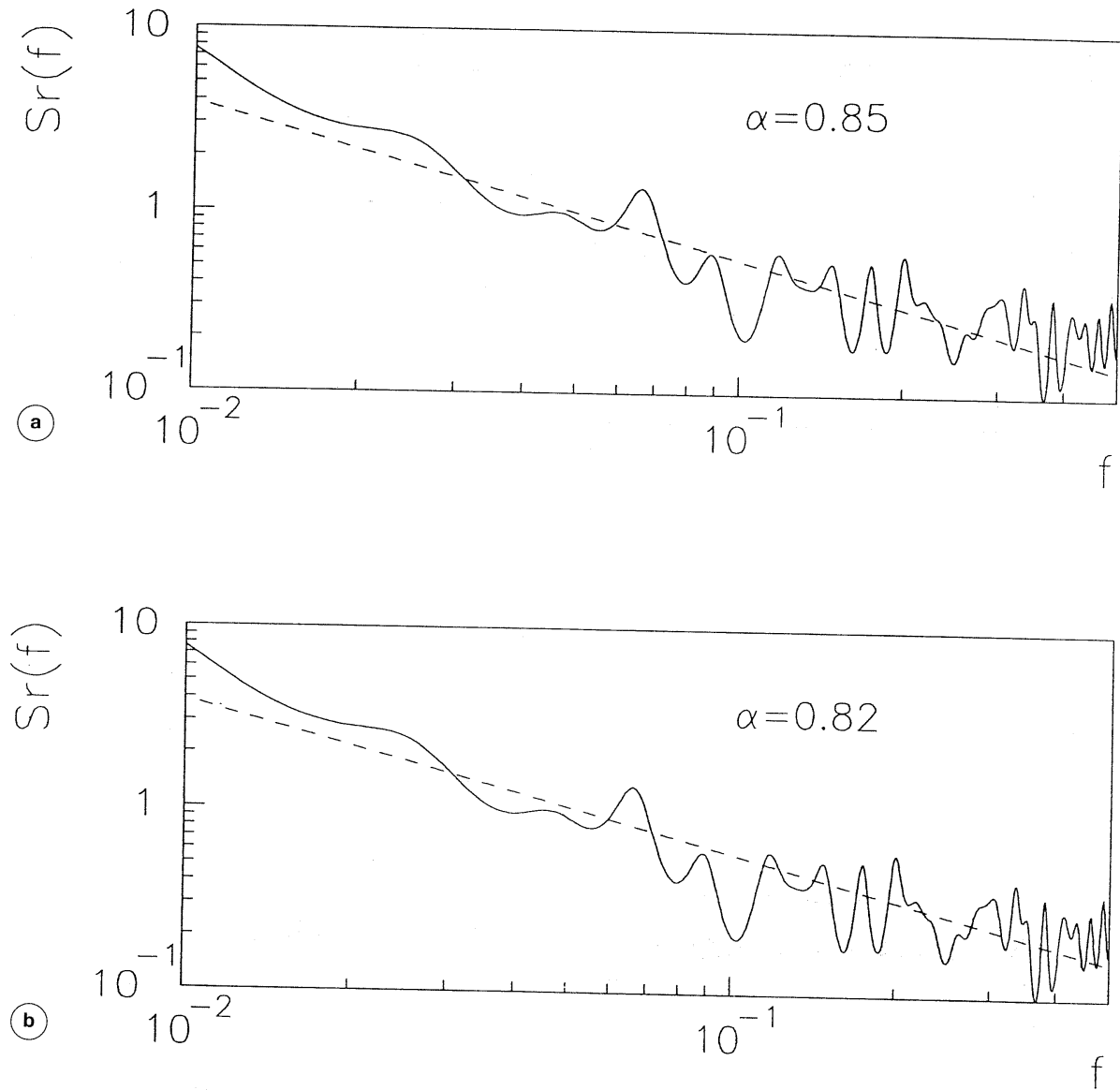
Fig. 7a,b. Power spectra of daily self-potential time series on $\log -\log$ plots. The two graphs (a) and (b) are re-
lated to the different dipole orientations.

these processes follow a linear behaviour on a $\log -\log$ scale.

To compute the power spectra we used the well known algorithm of the maximum entropy (Burg, 1968). Power spectra of daily geoelectrical time series showed on double log-scales a linear behaviour with angular coefficient close to 1 (fig. 7a,b). The temporal dynamics of daily self-potential time series was effectively a realization of a $1 / f$ noise, giving us a signature of self-organized criticality. To complete our analysis the power spectra of each sub-sample were computed. Four examples are plotted in fig. 8, and also in this case we noted the presence of $1 / f$ noise.

Our findings are not sufficient to consider the geoelectrical phenomena the output of a self-organized system, but all the results allow us to consider the system governed by infinite dimensional dynamic equations. In this case the classical approach based on the «mean field theory» or on the perturbative methods fails. In nature some dynamical systems act in a more concerted way. Different variables support each other in a way which cannot be understood studying the individual isolated constituents. This result has great implications on the earthquake prediction problem. 

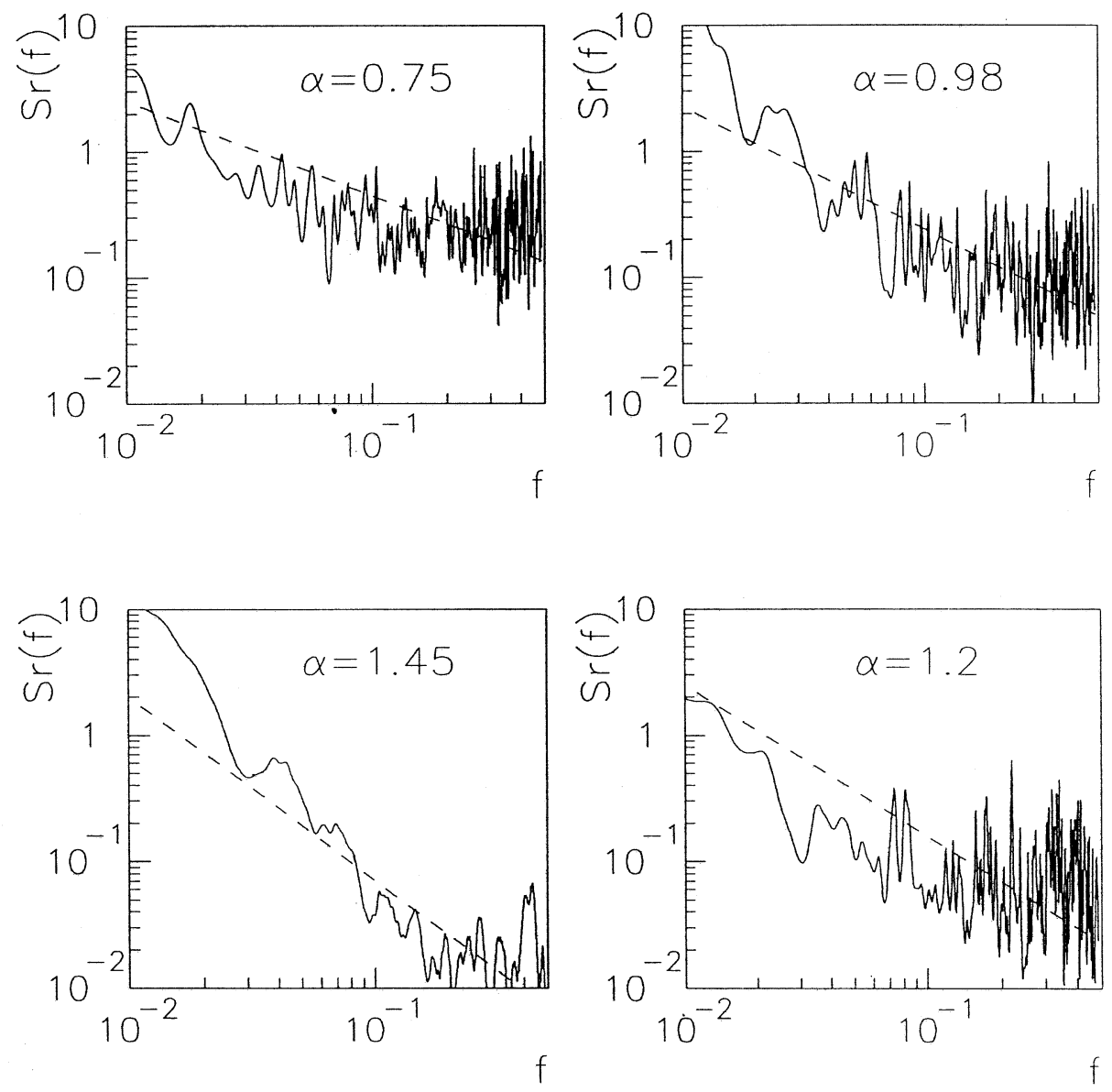

Fig. 8. Examples of power spectra obtained from different portions of self-potential data. The sampling interval is $\Delta t=5$ min and each sub-set is built up with 2880 data points.

\subsection{Implications on the earthquake prediction problem}

In this sub-section we point out the problems underlying the use of the electrical precursors in earthquake prediction. As an example, the correlation between the geoelectrical time series recorded by the Tito station and the seismic sequences is discussed. The ambiguity regarding the efficiency of precursory phenomena of electrical nature is due to the complicated interactions between all the parameters governing the phenomena. It is a hard task to approach the earthquake prediction problem studying the correlation between a single precursor of electrical nature and the incoming earthquake: our findings allow us to conclude the phenomenon cannot be predicted on the basis of a few prognostic equations. This is a possible explanation for many ambiguous results obtained with electrical precursory phenomena especially when we analyze the correlation between anomalous electrical patterns and seismic events with low magnitude $(M<3.5)$. 
To support our conclusions an experimental example is discussed: we showed the ambiguous results we obtained when we correlated the seismic events recorded in the area around the Tito station and the self-potential anomalies. First, the geoelectrical time series were filtered: long period components related to meteoclimatic effects were removed and all the data were normalized in mean and variance (Di Bello et al., 1994).

As regards the seismic sequences, since the earthquake precursors are variations in geophysical fields caused by a local earthquake preparation process, only earthquakes that could be responsible for strain effects in the investigated area must be selected. The stress field produces cracks on the rock volumes triggering fluid pressure variations. As a result of this process we have an underground charge motion and, subsequently, we observed anomalies in the electrical field on the surface only if the preparation region was near the measuring station.

It is necessary to discriminate the useful events (i.e., earthquakes responsible of significant geophysical variations in the rock volume of the investigated area) from all the seismic sequences which occurred in the area surrounding Tito measuring station.

To this purpose an empirical formula introduced by Dobrovol'skiy (Dobrovol'skiy et al., 1979; Dobrovol'skiy, 1993) was used:

$$
r=10^{.43 M}
$$

where $M$ is the magnitude and $r(\mathrm{~km})$ the radius of the area in which the effects of the earthquake are detectable. From the seismic events listed in the catalogue of the National Institute of Geophysics we considered only the earthquakes with $r$ greater than the distance between the epicentre and the measuring station. Obviously this technique is based on an empirical methodology, the situation is even more complex and the behaviour of precursors at different points of the preparation region may be substantially different.

Figure 9a-c reports the residual geoelectrical time series, characterized by zero mean and unit variance, and the selected earthquakes, while the dashed lines correspond to the limits of a $2 \sigma$ interval. Figure 9 a depicts the residual time series of the first year of monitoring activity. During the period May 1991-June 1991 a $M=4.7$ earthquake occurred and we also observed a large number of aftershocks. In the same period we picked out from the time series a very significant anomaly (abnormal values greater than $2 \sigma$ ) and observed a very sharp effect. The abnormal values started three days before the earthquake and we observed many scattered values in the coseismic and postseismic periods. In the subsequent period only a few earthquakes with very low magnitude occurred and we did not observe significant anomalous patterns in the residuals time series. Only few sparse residual data were over the threshold.

As depicted in fig. 9b, on May 8, 1992 a $M=3.7$ earthquake occurred near the Tito station and a sequence of anomalous values was clearly visible during the same period. The curve of experimental data shows a rapid increase since May 2 and it is clearly recognizable for many days during the coseismic and postseismic phases. During the subsequent period we had some earthquakes with magnitudes ranging from 3 to 3.5 , but we did not observe very significant extreme values in the residual time series.

As we can see in fig. 9c, during the first week of July and September we had few extreme values (i.e., the number of consecutive abnormal values is less than 3) and during $\mathrm{Au}-$ gust a $M=3.4$ earthquake occurred. In this last period a correlation between these values and the seismic events is not reliable, and it is an hard task to distinguish the preseismic and postseismic effects.

The last correlations are examples of the ambiguous results we obtain when we study the electrical precursory phenomena: an anomalous pattern can be detected but we do not have a physical basis to give a firm result. We have only two anomalies clearly detected, in other cases we have earthquakes without anomalies and vice versa. This effect is more evident when the magnitude of the seismic events becomes lower.

In our opinion, the electrical anomalies are really linked with earthquake activity but, ac- 

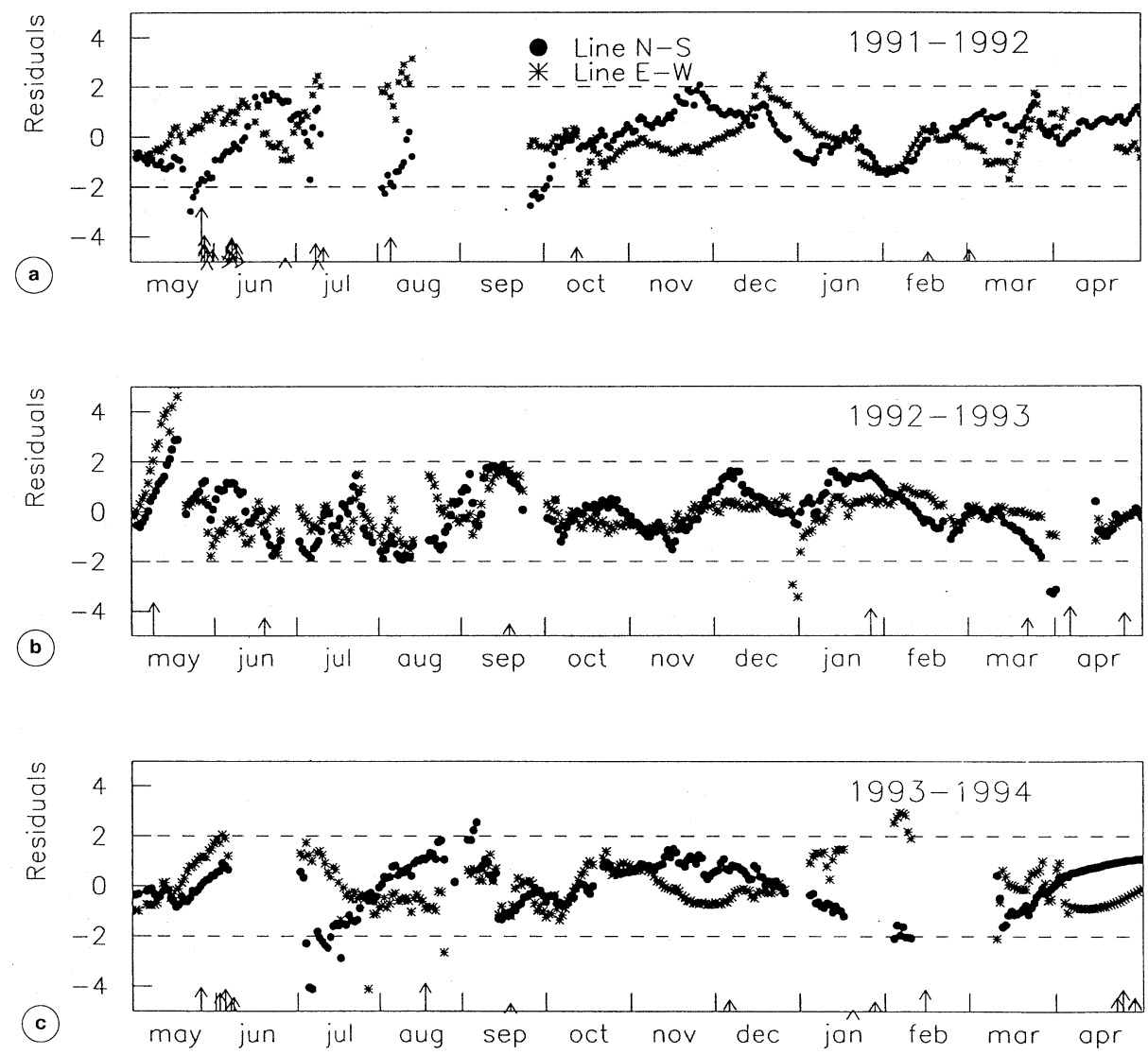

Fig. 9a-c. Residual self-potential time series and earthquakes occurred in the investigated area during the period May 1991-April 1994. The dashed lines delimit the band $\pm 2 \sigma$.

cording to the short-term predictability analysis described in this paper, the ambiguity, especially for earthquakes with low magnitude, is inside the physical process that produces the electrical anomalies, the dynamical laws governing the process being infinite dimensional.

\section{Conclusions}

The predictability of the geoelectrical time series has been evaluated using two possible forecasting approaches: global autoregressive approximation and local autoregressive approximation. For the daily mean time series our findings are that the global (linear) approach is always superior to the local one: the geoelectrical time series are truly stochastic. Power spectra of the residual time series follow a $f^{-\alpha}$ law, $f$ being the frequency, so they exhibit the typical behaviour of the broad class of $1 / f$ noises that are typical fingerprints of a self-organized system. These dynamic characteristics have important implications on the predictability of the series. As opposed to the chaotic case, we conclude the phenomenon at hand cannot be predicted on the basis of a few prognostic equations (whether linear or nonlinear), since its predictability is intrinsically limited. We do not have the possibility to de- 
scribe our geoelectrical time series with a low number of parameters, meaning that it is very difficult to approach the earthquake prediction problem using a single electrical precursor. In the near future we will develop a multiparametric monitoring array to analyse the space and time patterns of precursory phenomena in seismic areas.

\section{REFERENCES}

BAK, P., C. TANG and K. WiEsENFELD (1988): Self-organized criticality, Phys. Rev. A, 38 (1), 364-374.

BALDERER, W. and G. MARTINELLI (1994): Geochemistry of groundwaters and gases occurring in the November 23, 1980 earthquake area, Environ. Geochem., 16 (suppl.), 147-164.

Boschi, E., D. PAntosti and G. VAlensise (1994): L'identificazione geologica delle faglie sismogenetiche, Le Scienze, 310, 36-46.

Box, G.E.P. and G.M. Jenkins (1976): Time Series Analysis (Holden-Day, S. Francisco), pp. 350.

BURG, J.P. (1968): A new analysis technique for time series data, presented at Nato Advanced Study Institute of Signal Processing, Enschede, The Netherlands. Reprinted (1978) in Modern Spectrum Analysis, edited by D.G. CHILDERS (IEEE Press, New York), 42-48.

BurTON, P.W. (1985): Electrical earthquake prediction, Nature, 315, 315-317.

CASDAGLI, M. (1992): Chaos and deterministic versus stochastic non-linear modelling, J.R. Statist. Soc. B, 54, 303-328.

Cuomo, V., C. Serio, V. Crisciani and A. Ferraro (1994): Discriminating randomness from chaos with applications to a weather time series, Tellus, 46A, 299-313.

Cuomo, V., G. Di Bello, V. Lapenna, M. Macchiato and C. SERIO (1996): Parametric time series analysis of extreme events in electrical earthquake precursors, Tectonophysics, 262, 159-172.

Di Bello, G., V. Lapenna, C. Satriano and V. TramuTOLI (1994): Self-potential time series analysis in a seismic area of Southern Apennines: preliminary results, Annali di Geofisica, 37 (suppl.), 1137-1148.

Di Bello, G., V. Lapenna, M. Macchiato, C. SatriaNo, C. Serio and V. Tramutoli (1996): Parametric time series analysis of geoelectrical signals: an application to earthquake forecasting in Southern Italy, Annali di Geofisica, 39, 11-21.

DOBROVOL'SKIY, I.P. (1993): Analysis of preparation of a strong tectonic earthquakes, Phys. Solid Earth, 28 (6), 481-492.

DoBRovol'skiY, I.P., S.I. ZuBKov and V.I. MiACHKIN (1979): Estimation of the size of earthquake preparation zones, PAGEOPH, 117, 1025-1044.

Drahos, J., M. Puncochar, E. Nino and C. Serio (1995): Finding chaos in experimental time series: the case of two-phase flow, Il Nuovo Cimento, 110 B(12), 1415-1428.
Eftaxias, K., F. Vallianatos and J. Polygiannakis (1993): Evaluation of the success of earthquake prediction beyond chance, Prakt. Akad. Athenon, 68, 287-305.

FARMER, J.D. and J.J. SIDOROWICH (1987): Predicting chaotic time series, Rev. Phys. Lett., 59, 845-848.

Hadjicontis, V. and C. Mavromatou (1994): Transient electric signals prior to rock failure under uniaxial compression, Geophys. Res. Lett., 21 (16), 1687-1690.

JoAniuX, L., S. Lallemant and J. POzZI (1994): Changes in the permeability, streaming potential and resistivity of a claystone from the Nankai prism under stress, Geophys. Res. Lett., 21 (2), 149-152.

KenNel, M.B. and S. IsABelle (1992): Method to distinguish possible chaos from colored noise and to determine embedding parameters, Phys. Rew. A, 6, 31113118.

Lapenna, V., M. Macchiato, D. Patella, C. Satriano, C. Serio and V. TRAmutoli (1994): Statistical analysis of non stationary time voltage records in geoelectrical prospecting, Geophys. Prospect., 42, 917-952.

Mulargia, F. and P. GasperinI (1992): Evaluating the statistical validity beyond chance of «VAN» earthquake precursors, Geophy. J. Int., 111, 32-44.

Nishizava, O., X. LeI and T. NAGAO (1994): Hazard function analysis of seismoelectric signals in Greece, in Electromagnetic Phenomena Related to Earthquake Prediction, edited by M. HAYAKAWA and Y. FuJINAWA (TerraPub, Tokyo), pp. 98.

Nomikos, K., M. Bakatsakis, D. Paterakis, T. KogioNis, S. Sideris, B. Zaxaropoulos, C. Cristou, I. Kaliakatsos and F. Vallianatos (1994): Development of a telemetric system for observation of radioemission associated with earthquakes in Crete Island, in Proceedings of XXIV General Assembly of European Seismological Commission, 1112-1120.

Pantosti, D. and G. VAlensise (1990): Faulting mechanism and complexity of the November 23, 1980, Campania Lucania earthquake, inferred from surface observations, J. Geophys. Res., 95 (B10), 15329-15341.

PARK, S.K. and D.V. FitTERMAN (1990): Sensitivity of the telluric monitoring array in Parkfield, California, to changes of resistivity, J. Geophys. Res., 95 (B10), 557-571.

Press, W.H., B.P. Flannery, S.A. TeUKOlsky and W.T. VetTERLing (1986): Numerical Recipes (Cambridge University Press, London), pp. 818.

Raleigh, B., G. Bennet, H. Craig, T. Hanks, P. MolNAR, A. Nur, J. Savage, C. Scholz, R. Turner and F. WU (1977): Prediction of the Haicheng earthquake, EOS, Trans. Am. Geophys. Union, 236-272.

RIKITAKE, T. (1988): Earthquake prediction: an empirical approach, Tectonophysics, 148, 195-210.

SERIO, C. (1992): Discriminating low-dimensional chaos from randomness: a parametric time series modelling approach, Il Nuovo Cimento, 107B (6), 681-701.

SERIO, C. (1994a): Detecting chaos in time series, in Fractals in the Natural and Applied Sciences, edited by M.M. NovaK (Elsevier, North Holland), 371-383.

SERIO, C. (1994b): Autoregressive representation of time series as a tool to diagnose the presence of chaos, Europhysics Letters, 27, 103-108. 
SornetTe, A. and D. SornetTE (1989): Self-organized criticality and earthquakes, Europhysics Letters, 9, 197-202.

SornetTe, A. and D. Sornette (1990): Earthquake rupture as a critical point consequences for telluric precursors, Tectonophysics, 179, 327-334.

Sugihara, G. and R.M. MAY (1990): Nonlinear forecasting as a way of distinguish chaos from measurement error in time series, Nature, 344, 734-741.

TEISSEYRE, R. (1997): Generation of electric field in an earthquake preparation zone, Annali di Geofisica, 40, 297-304 (this volume).

Tsonis, A.A. and J.B. ElSNER (1992): Nonlinear prediction as a way of distinguish chaos from random fractal sequences, Nature, 358, 217-220.

TurcotTe, D.L. (1992): Fractals and Chaos in Geology and Geophysics (Cambridge University Press, London).
VArotsos, P. and K. Alexopoulos (1984a): Physical properties of the variations of the electric field of the Earth preceding earthquake, Tectonophysics, 110, 99-125.

VArotsos, P. and K. Alexopoulos (1984b): Physical properties of the variations of the electric field of the Earth preceding earthquakes 2: Determination of epicentre and magnitude, Tectonophysics, 110, 99-125.

VARotsos, P., K. Alexopoulos and M. LAZARIDOU (1993): Latest aspects of earthquake prediction in Greece based on seismic electric signals, Tectonophysics, 224, 1-39.

Voss, R.F. (1989): Random fractals: self-affinity in noise, music, mountains and clouds, Physica D, 38, 362-371.

WALES, D.J. (1991): Calculating the rate of loss of information from chaotic time series by forecasting, Nature, 350, 485-488. 